\title{
Noms collectifs humains : nomination et prédication
}

Human Collective Nouns: Naming and Predication

Michelle Lecolle

\section{(2) OpenEdition}

1 Journals

Édition électronique

URL : http://journals.openedition.org/aad/2208

DOI : 10.4000/aad.2208

ISSN : $1565-8961$

Éditeur

Université de Tel-Aviv

Référence électronique

Michelle Lecolle, " Noms collectifs humains : nomination et prédication », Argumentation et Analyse du Discours [En ligne], 17 | 2016, mis en ligne le 15 octobre 2016, consulté le 23 septembre 2019. URL : http://journals.openedition.org/aad/2208; DOI : 10.4000/aad.2208

Ce document a été généré automatiquement le 23 septembre 2019

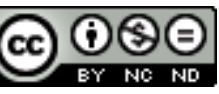

Argumentation \& analyse du discours est mis à disposition selon les termes de la licence Creative Commons Attribution - Pas d'Utilisation Commerciale - Pas de Modification 4.0 International. 


\title{
Noms collectifs humains : nomination et prédication
}

Human Collective Nouns: Naming and Predication

\author{
Michelle Lecolle
}

\section{Introduction}

$1 \mathrm{Si}$, dans une perspective énonciative et discursive, toute nomination est porteuse d'enjeux pragmatiques et de points de vue - en termes d'axiologie ou de représentation -, on conviendra néanmoins que les moyens et les effets mêmes diffèrent selon les formes linguistiques à l'œuvre. Je m'intéresserai dans cet article à ce que, dans leur spécificité, les noms collectifs (Ncoll) rendent possible. Je me propose donc de situer les « enjeux socio-politiques » de la nomination dans le cadre d'une analyse sémantique et discursive plus générale portant sur la nomination de groupes humains par le biais de Ncoll. Il s'agit, à partir des propriétés sémantiques des Ncoll d'envisager leur implication en termes de potentialités discursives, qui les rendrait aptes à jouer un rôle, en tant que tels, dans le débat public. Mais l'étude se limitera aux seuls noms collectifs humains (Ncoll-H), incontestablement plus adéquats pour les questions touchant le politique et le social - la vie en société -, plus pertinents pour la thématique du numéro et, par ailleurs, plus nombreux et variés.

2 Une des caractéristiques des Ncoll est de rassembler par la dénomination une pluralité d'individus, sous un label au singulier. En elle-même, cette caractéristique sémantique est porteuse d'enjeux, et en particulier lorsqu'il s'agit d'humains: en effet, s'il existe bien, pour les non-humains, des groupes en quelque sorte à l'état naturel (un essaim, une forêt par exemple), ce rassemblement ne va pas de soi quand il s'agit des sociétés humaines. De fait, en dehors des cas où le Ncoll désigne un rassemblement conjoncturel (foule, assemblée), les rassemblements et modes de rassemblement d'humains que dénote le lexique des Ncoll-H renvoient plus largement à des construits culturels et sociaux (voir des exemples aussi divers que peuple, liste (électorale), gouvernement, magistrature, opposition, tribu). Dès lors, nommer par un Ncoll-H relève nécessairement 
d'un angle d'attaque, plus ou moins partial selon les cas. Par-delà ce noyau commun, la signification lexicale de certaines catégories de Ncoll-H ou de tel ou tel d'entre eux est susceptible de porter une orientation discursive voire argumentative: le cas le plus immédiat est celui des Ncoll-H axiologiques (élite, gratin, clique, racaille), mais d'autres méritent attention (voir l'évaluation négative de groupuscule, comparé à mouvement, plus flou et plus neutre; de milice comparé à police, garde (républicaine) plus neutres). Enfin interviennent d'autres paramètres, non principalement lexicaux, qui renvoient d'une manière générale à la manière dont le Ncoll-H est employé, dans les énoncés, et au sein des textes.

3 À travers ces différents niveaux potentiels, on est amené à rapprocher nomination et prédication, tant ces actes de langage apparaissent finalement proches et intriqués. Alors que le rapport entre prédication et argumentation va relativement de soi - dire quelque chose d'un sujet, c'est déjà orienter, donc argumenter à son propos -, il n'en est pas de même a priori avec la nomination, mais c'est précisément ce «chainon manquant » que la démarche cherche à reconstituer.

4 Comme le soulignent Perelman et Olbrechts-Tyteca, «le choix des prémisses et leur formulation [...] ne sont que rarement exempts de valeur argumentative » $(1970: 84)$, et l'avertissement vaut bel et bien pour la nomination, ici la nomination de réalités (ou supposées réalités) par un Ncoll-H. Pour autant, je ne considérerai pas cette nomination sous l'angle d'une éventuelle "visée argumentative », mais, selon la distinction posée par Amossy (2000) et reprise dans Amossy et Koren (2004), comme possédant bien une "dimension argumentative", en ce sens qu'elle est à même de "modifier les représentations de l'interlocuteur et de lui faire partager une vision des choses » ou, du moins, «[d'orienter] sa réflexion ou son regard ». Je ne m'intéresserai donc pas à une éventuelle intention argumentative ou manipulatrice à travers un énoncé, ni même, du moins directement, à l'argumentation en tant que telle ${ }^{1}$, mais davantage aux enjeux potentiels de la sémantique des Ncoll-H et de leurs emplois. Mes exemples proviennent d'énoncés et de fragments de textes contemporains, principalement médiatiques mais non nécessairement centrés sur des questions politiques ${ }^{2}$ : en effet, je suppose que les enjeux socio-politiques ou politiques des Ncoll-H et plus généralement de la nomination dépassent largement le cadre de ces thèmes. Je suppose également que ces enjeux peuvent être ouverts, déclarés, ou au contraire souterrains - échappant aux énonciateurs. Certaines observations apparaissent en ce sens dans l'article. La recherche française sur les Ncoll traite généralement ceux-ci sous l'angle de la référence, et, à ma connaissance, le versant prédicatif n'a jusqu'à maintenant pas été abordé. En tirant mes observations d'une recherche plus générale sur 275 Ncoll-H, c'est ce versant que je me propose d'explorer en m'intéressant au lien qu'on peut établir entre le potentiel que présente la prédication - en termes de qualification, classification ou identification -, et les enjeux que recèlent les Ncoll-H.

Dans une première partie, je m'attacherai à discuter le lien entre nomination et prédication, à partir de faits lexico-syntaxiques et de certaines structures mettant en œuvre, de manière implicite, la prédication. Dans une deuxième partie, je présenterai les traits principaux constituant la sémantique des Ncoll-H, en me centrant sur ce qui, les concernant, est potentiellement négociable, discutable, problématique, sensible, bref porteur d'enjeux, dans le référent supposé ou dans le signifié du Ncoll-H questions de constitution du groupe, d'appartenance et d'identité, axiologie. Dans une 
troisième partie, je reviendrai sur la prédication, cette fois explicite, par le biais des structures attributives avec Ncoll-H, sur la base de mes collectes d'exemples.

\section{Préalables théoriques et terminologiques}

6 J'emploie le terme de "nomination" pour désigner l'acte de langage qui consiste à sélectionner en discours une forme linguistique nominale pourvue de sens (signe, expression) pour renvoyer à un référent ${ }^{3}$ - que cette saisie soit directe ou non, et que le référent lui-même soit simple ou complexe, composite, délimité ou flou, ou encore imaginaire, ou pure création discursive. En regard de "nomination », j'emploie, à la suite de Kleiber (2001), le terme de "dénomination» avec ses deux acceptions: (1) forme lexicale codée appariée à un référent ainsi que (2) l'acception processuelle correspondant à l'emploi de cette forme codée. En résumé, les deux termes «nomination » et « dénomination » sont pour moi complémentaires, mais ne se situent pas au même niveau : "dénomination » renvoie à ce qui est codé, qu'il s'agisse d'une forme lexicale ou de l'emploi de cette forme, alors que «nomination", plus général, correspond à l'acte de langage de nommer, quelle que soit la forme employée et le référent.

\section{Nomination et prédication}

7 Alors qu'on oppose généralement les actes de référence - ici «nomination ${ }^{4}$ » - et de prédication, tests à l'appui (Charolles 2002), certains faits linguistiques montrent qu'il est pertinent de reconsidérer cette opposition en l'atténuant. J'aborde ces questions à travers les travaux de plusieurs auteurs.

8 Selon la définition classique, prédiquer c'est "dire quelque chose d'un sujet ». Cette conception, qui confond la prédication avec l'assertion, s'accompagne généralement, comme le remarque Sériot, d'une bipartition qui superpose, d'un côté, nom, expression de la référence, sujet et, de l'autre, verbe et prédicat, la prédication elle-même - qui correspond en grammaire à la proposition - étant le composé des deux. Selon cette conception, remarque encore Sériot, «les noms ne font que nommer des objets [...] naturels, [...] connaissables directement" (1993: 244). Or, il suffit de découpler prédication et assertion et, par exemple, d'examiner, comme le fait l'auteur pour le français et le russe, les nominalisations de procès pour discerner la présence de la proposition au sein même du syntagme nominal (SN) - «il donne son sang/le don de son sang». Ces faits sont certes connus ${ }^{5}$, mais la description (dont je n'extrais ici que quelques éléments) permet de réaliser tout ce que la présence de la prédication dans le domaine nominal et le SN a comme corrélats en termes de qualification et d'évaluation, d'implicite et de présupposé, en d'autres termes de préconstruit, les nominalisations pouvant «être la mémoire du déjà-dit aussi bien que du dicible» (p. 257). Grammaticalement et sémantiquement circonscrits, les faits pris pour exemple (nominalisations de procès) sont néanmoins d'un tout autre ordre que ceux que j'examine : il ne s'agit pas de Ncoll, pas de noms d'objets ou de personnes, pas non plus de noms évaluatifs.

9 Mais le caractère prédicatif de SN est en réalité tout à fait courant. Je présente dans ce qui suit quelques phénomènes dans lesquels cette prédicativité est particulièrement 
visible - il va sans dire que l'exhaustivité est ici impossible. Voici quelques exemples, dont certains avec des Ncoll-H.

Le caractère prédicatif de $\mathrm{SN}$ se rencontre notamment dans les anaphores ${ }^{6}$ : la question apparait, directement ou de manière plus implicite, à travers les études sur l'anaphore définie $^{7}$ (Van Peteghem 1998, Reichler-Béguelin 1998), ou, plus particulièrement, sur l'anaphore démonstrative ${ }^{8}$ (Gary-Prieur 1998, Reichler-Béguelin 1995, Wiederspiel 1995). On parle parfois d'anaphore prédicative (Schnedecker 2006) - voir plus loin. Retenons en résumé quelques éléments :

- selon les cas, les SN employés anaphoriquement identifient, (re-)classifient, ou qualifient le référent (l'objet-de-discours) présenté auparavant; ils peuvent aussi apporter des informations spécifiantes et/ou identifiantes sur cet objet-de-discours (Reichler-Béguelin 1998). La signification du nom de ces SN anaphoriques est ici d'une importance primordiale ;

- tout en référant, le SN renomme et, en fait, prédique, par le biais d'une prédication implicite, présupposée, du type "est-un " (qualifiant, classifiant, identifiant) - une relation-être ${ }^{9}$ : ainsi, en (1) ci-dessous, Agnieszka Holland "est-une " cinéaste (identification) ; en (2) Marie « est-une » femme charmante (qualification) ; (3) est plus complexe, où Jérôme Monod «est-un" absent, «est-un » illustre fantôme (qualification) et, en même temps, l'absent, l'illustre fantôme "est " Jérôme Monod (identification).

(1) Agnieszka Holland [...]. La cinéaste a gardé un souvenir ému [...] de cette histoire

(Le Magazine Gaumont, exemple de Van Peteghem 1998)

(2) En épousant Marie, tu as fait le malheur d'une femme charmante (exemple de Corblin, rapporté par Van Peteghem 1998)

(3) Le procès d'Alain Carignon [...]. Son supposé corrupteur reste invisible. [...] Cet absent [...], le nom de cet illustre fantôme ? Jérôme Monod [...] (Le Canard Enchainé, exemple de Reichler-Béguelin 1998).

11 Les exemples (4), (5) et (6) appartiennent à mes relevés de Ncoll-H. Respectivement, en (4), la pluralité de «les pigeons" « est-un» mouvement très hétéroclite d'entrepreneurs ; en (5), Boko Aram «est-une » organisation salafiste. Dans les deux cas, les SN avec Ncoll-H sont classifiants, et les énoncés auxquels ils appartiennent ont une fonction didactique. Quant à secte appliqué à Boko Aram, il est qualifiant ou classifiant selon le sens qu'on lui attribue: axiologie (négative) ou catégorie sociologique appliquée à la religion ? Le glissement entre les deux se fait aisément.

(4) L'opération de charme à l'attention du monde de l'entreprise s'est poursuivie hier en direction des "pigeons ", venus nombreux à l'Élysée [...]. Ce mouvement très hétéroclite d'entrepreneurs [...] (Libération, 29/04/2013).

(5) [...] Il s'agit de s'attaquer à Boko Aram par le nerf de la guerre. [...]. Car l'impressionnant arsenal de guerre dont la secte dispose, le nombre de combattants qu'elle entretient et les moyens de communication sophistiqués qu'elle utilise sont la preuve que cette organisation salafiste bénéficie de fonds secrets (Courrier International, 15-21/05/2014).

Enfin, si, comme pour les autres, l'on peut gloser (6) en «la Manif pour tous » « estune " nébuleuse, peut-on réellement trancher, avec ce nom, entre classification et qualification, ou encore identification?

(6) [À propos de «la Manif pour tous »] Ce qui frappe dans cette nébuleuse, c'est l'osmose entre tous les courants (Politis, 25 avril 2013).

Les exemples avec Ncoll-H présentent des propriétés particulières liées à la pluralité voir (4) -, mais ils sont comparables aux autres, pour ce qui m'occupe : la reprise 
anaphorique prédicative d'un objet de discours. Quoi qu'il en soit, on retrouve dans les cas d'anaphoriques le préconstruit de la prédication, déjà signalé avec les nominalisations de verbes, et c'est bien ce qui rapproche, par-delà leurs différences, les deux cas mentionnés dans cette section: procès d'un côté - les nominalisations (donner/le don) -, qualification, classification ou identification de l'autre - les reprises anaphoriques (ce mouvement, la secte).

14 En dehors de ces deux cas, d'autres faits syntactico-sémantiques permettent d'illustrer et d'expliquer les enjeux discursifs de la nomination par la présence de la prédication au sein du SN : il s'agit notamment des phénomènes généralement décrits en termes de "prédication seconde ", en particulier dans les appositions composées d'un SN - (7a) avec l'imbécile, et (7b) avec le Ncoll-H formation - et les SN complexes en [N1 (de) N2] comportant un nom tête qualifiant - imbécile, crétin en (8a), lie en (8b) - ou classifiant collectif en $(8 \mathrm{c})^{10}$.

(7a) Jean, l'imbécile, a cassé la tasse (exemple de Milner 1978)

(b) La ferveur contagieuse de Fauve, une des formations les plus originales du moment (Télérama, 17/04/2013, pages musique)

(8a) L'imbécile/ le crétin/ cet imbécile/ ce crétin de Jean a cassé la tasse (à partir d'exemples de Milner)

(8b) [...] une lie d'aventuriers, de spéculateurs, de fraudeurs (Van der Mersch, cité par Lammert 2010)

(8c) [...] La mobilisation citoyenne des membres du collectif Anonymous qui ont manifesté [...] en face du Celebrity Center pour dénoncer les dangers de la Scientologie [...] (site Anonymes France ${ }^{11}$ ).

Ces exemples sont similaires du point de vue, très général, de la présence de la prédication dans le SN, mais on ne manque pas de remarquer au passage la différence de force et de fonction discursive que représentent les termes classifiants (collectif, formation, organisation) vs qualifiants (imbécile, crétin, lie) - je reprends ces questions à propos des Ncoll-H en $§ 2.3$ et en $\S 3$.

Au-delà, sont potentiellement concernés tous les cas de nomination non orthonymique - non strictement dénominative, donc - dans lesquels, pour être effective et réussie, la nomination nécessite un apprentissage, une explication, une interprétation préalables. La métaphore, dans la conception développée par Ricœur, apparaît ici comme le modèle, le type par excellence, dans la mesure où elle a fondamentalement un caractère prédicatif : selon Ricœur, plutôt qu'en termes de "dénomination déviante ", elle doit être caractérisée comme une "prédication impertinente» (1975: 8). Il dit encore :

[...] la dynamique de la métaphore in absentia n'est révélée que par la métaphore in praesentia, où c'est l'interaction entre tous les termes de l'énoncé qui motive la substitution d'un terme présent à un terme absent (247, en note).

Si on laisse de côté le principe sémantique de la métaphore - ressemblance, similitude -, les faits de nomination qui m'occupent sont, comme pour ce trope, à interpréter (in absentia) sur la base d'une ou de prédication-s (in praesentia), reconstituable-s ou antérieure-s, restant à titre de présupposé dans la désignation occurrente.

Considérons (9), qui illustre plusieurs des points abordés précédemment :

(9) Et puis Ramzan Kadyrov a obtenu des moyens: les millions donnés par le Kremlin, ainsi qu'une aide des Émirats Arabes Unis. Il a beaucoup reconstruit, favorisé le retour du religieux en édifiant d'immenses mosquées, imposé la charia. Cette manne ne bénéficie évidemment pas à tout le monde. Comme en Russie, elle va à une caste, à des happy few du clan ou de ses proches. On croise les hommes de 
cette nomenklatura dans les rues de Grozny au volant de leurs grosses voitures [...]. Et, pendant ce temps, plus de $40 \%$ de la population tchétchène est au chômage (interview de la journaliste Manon Loizeau, à propos d'un documentaire sur la Tchétchénie, Télérama, 25/02/2015).

Dans cet exemple ${ }^{12}$, on trouve plusieurs nominations reposant sur ce principe prédicatif, avec ou sans métaphore. Selon moi, les Ncoll-H caste et, peut-être, nomenklatura ${ }^{13}$ (qui reprend caste en anaphore prédicative), ainsi que le nom manne (en anaphore prédicative) sont des nominations métaphoriques: le référent $\mathrm{du}$ groupe constitué des proches de Ramzan Kadyrov est vu/considéré comme/comparé à une caste/une nomenklatura ; l'argent provenant des Émirats Arabes Unis est vu/considéré comme/comparé à une manne. Parallèlement, le Ncoll-H clan, supposé non métaphorique, trouve son interprétation dans les prédications antérieures du texte : en effet le régime décrit fonctionne bien sur la base d'une organisation en clans. Selon cette interprétation, le terme est factuel.

Cet exemple et les analyses qui précèdent débouchent cependant sur une question qui, elle non plus, n'est pas nouvelle, mais à laquelle il me semble difficile de donner une réponse définitive: quels seraient finalement les cas où l'on peut se passer d'une analyse prédicative ? Ou, dit en d'autres termes : dans le monde des humains, et, plus particulièrement, dans le monde des groupes et des organisations humaines, c'est-àdire, en fait, dans la vie politique et sociale, $y$ a-t-il vraiment des cas possibles d'orthonymie, c'est-à-dire de pure et simple dénomination ? Sur la base du contexte qui précède (9), ou pour qui connaît bien la situation décrite par l'article, le nom clan constitue sans doute une réponse positive à cette question - l'organisation en clans est, semble-t-il, un fait d'organisation de la vie politique dans certains pays. Mais les cas de ce type - appariement mot/chose - me paraissent plutôt l'exception que la règle. Il suffit d'ailleurs de mettre clan dans un contexte différent pour qu'il trouve matière à métaphore - voir « Émilie et son clan », commentaire de l'émission Secret Story sur TF1.

Comme on le sait, aucun mot n'est innocent, les orthonymes pas plus que les autres. Mais ce sont les modalités de cette (non-)innocence que je cherche à préciser pour les Ncoll-H. Dans ce qui suit, je tirerai parti de la réflexion présente pour la croiser avec ce qui tient aux propriétés des Ncoll-H.

\section{Noms collectifs humains : propriétés sémantiques et lexicales}

\subsection{Noms collectifs, Noms collectifs humains : individus et groupe constitué}

De manière très classique, on peut définir le Ncoll en français comme : « un nom qui, au singulier, dénote une entité composée d'un regroupement d'éléments » (Lammert 2010, Lammert et Lecolle 2014). Certaines définitions ajoutent que les éléments sont « de même catégorie », ce qui pose naturellement la question de ce qu'on peut considérer comme tel - cette question revient en filigrane dans ce qui suit (voir $\S 2.2$ et 2.3). Les Ncoll-H ajoutent aux traits des Ncoll (pluralité, regroupement) un trait humain, qui, pour la plupart d'entre eux, est en lien avec le mode de regroupement - ou la représentation qu'on en suppose: quelle différence, sinon, entre réseau, bande, mouvement ou groupuscule? 

poser, sous une dénomination, le rassemblement idéel d'individus potentiellement dispersés et divers. Si, comme le dit Frath (2010), "la dénomination ${ }^{14}$ possède [1']indéniable pouvoir démiurgique [de faire exister une chose pour nous] », elle la fait exister de manière spécifique pour les Ncoll, et la «chose» en question est de type particulier : tout à la fois un ensemble, c'est-à-dire une pluralité, et une unité, et c'est pourquoi j'ai introduit plus haut l'idée de "référent complexe, composite ", mais aussi de «flou». Ce premier niveau d'analyse relève de la question, en quelque sorte métaphysique, de l'existence même d'une entité, celle du groupe, question qui, naturellement, présente des enjeux discursifs, pour des raisons évoquées en introduction: pour des sociétés humaines, le regroupement même et le type de regroupement ne sont pas neutres. soit qu'ils mettent en lien ou en balance des individus et un groupe constitué - (10) à (13) -, soit qu'ils interrogent, voire contestent l'existence ou les modalités de ce groupe, dans une démarche explicitement métalinguistique - (14) à (17) -, notamment par le biais de la négation. Les deux catégories d'exemples peuvent d'ailleurs paraître artificiellement distinguées, dans la mesure où les traits descriptifs que je mentionne y apparaissent parfois mêlés - comme il arrive généralement dans des exemples attestés.

Pour plus de clarté, je signale en italique sous chaque exemple (10) à (13) les segments concernés :

(10) [Jacques Copeau, 1913]. Il a recruté onze jeunes comédiens, qu'il s'efforce maintenant de former. [...] À ses heures plus violentes, il parle même d'« endoctrinement ». Histoire de mieux faire saisir à la troupe naissante qu'elle doit obéir à d'autres lois que la recherche de l'effet [...] (Télérama, 07/13).

Onze jeunes comédiens / troupe naissante

(11) [Les auteurs abordent] la notion de peuple comme résultant de l'histoire d'un rapport de forces. [...] Que ce soit dans un élan « performatif » [...], comme, sur la place Tahrir au Caire, où les corps réunis dans la rue se "constituent en peuple " face au pouvoir (Politis, 6/4/2013, compte rendu de lecture "Qu'est-ce qu'un peuple? » La Fabrique, 2013).

Les corps réunis / peuple

(12) [élections municipales] À Molène, parler de liste serait exagéré. Plus qu'un groupe portant un programme, ce sont des candidatures individuelles qui s'additionnent. Dix-neuf personnes, qui acceptent, avec énergie ou à contrecœur, de porter la vie de la commune (Politis, 25/03/2014).

\section{Des candidatures individuelles / liste, groupe}

(13) À deux jours de la fin [du festival de Cannes], on tient la palme. Enfin, «on », une sorte de confrérie critique, informelle mais assez large, dont les membres non répertoriés et aléatoires se croisent plus ou moins régulièrement au Palais du Festival ou dans les rues de Cannes, et qui désormais échangent maints tweets au sortir des projections (Journal de Cannes, Christophe Kantcheff, Politis, 30/05/2013).

[Des] membres non répertoriés et aléatoires / confrérie critique. Voir aussi le modalisateur « une sorte de ».

Dans la série suivante, plus explicitement encore, est posée ou niée l'existence d'un groupe, et de tel ou tel type de groupe, sans que les éléments soient directement mentionnés. Qu'il y ait affirmation ou négation d'existence, on a affaire, sous couvert 
de description, à de véritables actes de langage, et ceci d'autant plus lorsque l'énonciateur est un dirigeant politique - (14) :

(14) «La société n'existe pas. Il y a seulement des hommes, des femmes et des familles " (Margaret Thatcher, Women's Own Magazine, 1987).

(15) « Uni dans la diversité », le peuple rom existe dans de multiples variantes en Europe (en titre, interview d'un chercheur dans Politis, 5/07/2012).

En (15), on comprend naturellement que si « le peuple rom existe ", c'est en tant que peuple, donc comme tout constitué, le titre jouant de la contradiction pour affirmer, proclamer un rassemblement (abstrait), à caractère identitaire. En (16), c'est aussi un tout (mouvement) qui, cette fois, est nié, mais le jugement métalinguistique négatif porte également sur la signification du Ncoll-H (" mouvement en tant que politique »).

(16) Il n'y a pas de mouvement rom. Même l'Union romani internationale n'est pas représentative de la totalité des Roms. Les Roms ne se laisseront jamais représenter par une partie d'eux-mêmes (corps du texte interview d'un chercheur, Politis, 5/07/2012).

31 En (17), où intervient également la signification politique de mouvance, l'adjectif modalisateur supposée a un ancrage équivoque : s'agit-il de discuter (1) l'existence d'une mouvance? (2) L'existence de l'ultra-gauche en tant que telle? Ou (3) mouvance comme qualificatif (péjoratif ?), appliqué à ce groupe que serait l'ultra-gauche?

(17) Cette supposée mouvance de l'ultra-gauche (presse, 2012)

On retrouve en (3) dans cette structure [N1 de (dét) N2] le rôle de la prédication discuté en $\S 1$. Selon moi, c'est bien la sémantique des Ncoll dans son fondement qui permet d'expliquer et de prévoir que ces noms soient discutables, polémiques dans leur relation à un référent, et, comme en (17), qu'ils puissent être sémantiquement ambigus.

\subsection{Noms collectifs humains, appartenance et identité}

Abordons à présent ce qui constitue un autre pan de la sémantique des Ncoll, humains en particulier, à savoir la question de l'appartenance au groupe et de son rapport à l'identité des membres ${ }^{15}$. En effet, si, comme dit plus haut, le Ncoll-H constitue par une dénomination un "rassemblement idéel d'individus potentiellement dispersés et divers ", on peut penser que, pas plus que le rassemblement d'individus, le gommage de leurs différences par la dénomination ne va de soi. On peut aussi penser, par ailleurs, que l'appartenance même de ces individus au (supposé) groupe a des conséquences en termes de représentation voire de qualification de ces individus. On a bien affaire ici à ce que Perelman et Olbrechts-Tyteca proposent de décrire en termes de «liaisons de coexistence qui unissent [...] un groupe aux individus » (1970:353).

Certains Ncoll-H sont à ce titre, et de différentes manières, plus «brûlants » que d'autres, c'est-à-dire qu'ils peuvent être l'enjeu d'assignations identitaires discutables, soit parce qu'elles sont d'ordre graduel et difficiles à trancher, soit aussi parce qu'elles croisent des questions politiques ou sociales. On peut comparer à cet égard les Ncoll-H orchestre et magistrature d'un côté et, de l'autre, droite, gauche dans le monde politique ou encore diversité et minorité employés comme désignateurs et catégoriseurs de groupes sociaux: "appartenir à un orchestre" est lié et même équivalent à "être musicien » - de même pour magistrature/magistrat -, et on peut supposer que cette équivalence ne pose pas problème; mais "appartenir à la droite/gauche", comme équivalent à « être de droite/gauche » est une appartenance qui peut se discuter. Enfin, 
que dire de l'appartenance posée par le Ncoll-H communauté dans [communauté + Adj catégorisant] (communauté kurde de Turquie, communauté musulmane) ? Ou diversité (Un préfet issu de la diversitée ${ }^{16}$ ) dans un contexte français, ou encore minorité - (18) ?

(18) Jusque là, Hrant Dink [Arménien en Turquie] n'avait jamais senti qu'il appartenait à une minorité. [...] le gouvernement turc taxe arbitrairement les minorités du pays, dont les Arméniens (Courrier International, numéro spécial sur la Turquie, mars-avril-mai 2014).

Dans le monde social, ces Ncoll-H sont tout aussi problématiques que les réalités qu'ils désignent... ou construisent, et s'ils le sont, c'est, d'une part, par le biais du lien entre appartenance et identité, d'autre part parce que, de par leur signification (minorité vs majorité, i.e. « le reste (de la société) »), ou de par la manière dont ils sont employés et réfèrent (diversité, communauté), ils renvoient à un " autre ", une altérité. La question du lien appartenance-identité est donc présente à travers le sens social de certains NcollH.

J'aborde à présent un autre niveau d'analyse, celui de la signification lexicale des NcollH.

\subsection{Signification lexicale: des Noms collectifs humains plus « sensibles » que d'autres}

Si aucun terme, collectif ou non, n'est (potentiellement) innocent dans le cadre des sociétés humaines, il ne parait toutefois pas judicieux de tout niveler, y compris au sein même des Ncoll-H; d'ailleurs, certains d'entre eux sont peu problématiques et, inversement, d'autres le sont intrinsèquement ${ }^{17}$. Il peut s'agir de leur signification lexicale ou de la manière, par exemple ironique, dont ils sont systématiquement employés. Il va sans dire que la plupart des Ncoll-H ont plusieurs acceptions, et qu'ils peuvent aussi, à l'instar des noms dans leur ensemble, être employés métaphoriquement - voir plus haut le cas de clan. Par ailleurs, il faut tenir compte de ce qui vient d'être signalé en 2.2, et qui, cette fois, ne concerne que les Ncoll (ici les NcollH) : la question de l'appartenance potentiellement problématique (ou pas) à tel groupe (orchestre vs droite / gauche vs diversité-s, minorité-s).

Voici, à partir de mon corpus de Ncoll-H, quelques propositions de différenciation tendant à donner un ancrage de sémantique référentielle et lexicale aux enjeux sociopolitiques de nomination par des Ncoll-H. Dans les limites de cet article, il me parait pertinent de saisir ces différenciations sous l'angle de l'évaluation ou de l'axiologie portée par les noms étudiés ${ }^{18}$ - même si ce choix est réellement une simplification, parfois trompeuse. Comme on le verra, cette axiologie est complexe, parfois indirecte, et notamment quand elle repose sur les enjeux, abordés $§ 2.1$ et 2.2 , de constitution du groupe, d'identité des membres, ou d'image du groupe sur la scène publique.

Plusieurs cas de figure ${ }^{19}$ :

\section{Ncoll-H « fonctionnels »}

39 Tout d'abord, mettons de côté les Ncoll-H a priori neutres d'un point de vue axiologique. Il s'agit de la grande majorité des 135 Ncoll-H que je classe comme "fonctionnels", c'est-à-dire pour lesquels le rassemblement des membres est basé sur un but commun, 
ou supposé tel. Voici quelques-uns d'entre eux, parmi lesquels certains rencontrés précédemment :

alliance, amicale, appareil, armée, association, bande, carmel, chorale, club, commission, confrérie, couple, équipe, famille, fanfare, formation, gouvernement, jury, ligue, liste (électorale), mouvance, mouvement, orchestre, organisation, parlement, personnel, police, quartet, réseau, service, syndicat, troupe.

Toutefois, certains autres Ncoll-H fonctionnels sont négativement marqués, pour des raisons liées au référent ou à la signification lexicale du nom - avec toutes les questions que pose cette distinction ${ }^{20}$. J'ai relevé cabale, gang, junte, lobby, mafia, milice, milieu, pègre, phalange, secte, pour lesquels la péjoration porte sur le référent collectif - et, par voie de conséquence, sur ses éléments. Comme pour phalange, pègre ou mafia, c'est l'activité supposée du groupe - polémique, illicite, dangereuse, occulte, attentatoire à la démocratie - qui est cause de la péjoration. Dans d'autres cas - bande, clan, coterie, faction, groupuscule, ligue -, le discrédit éventuel repose plutôt sur la signification du nom utilisé pour nommer (et qualifier par là-même) un référent collectif.

41 La frontière entre ces séries de Ncoll fonctionnels est parfois poreuse : on a vu que clan ou secte peuvent être purement factuels, et l'appréciation neutre ou négative (mais pas positive) de bande ou de ligue est labile.

\section{Ncoll-H intrinsèquement évaluatifs}

Inversement, certains Ncoll-H sont intrinsèquement évaluatifs. En conséquence, la nomination d'un groupe par un tel nom revient ipso facto à qualifier ce groupe - ces Ncoll-H sont prédicatifs. Ils sont péjoratifs (racaille, canaille, clique, coterie, vermine, lie), ou mélioratifs (crème, élite, gratin, sérail) - même s'il n'est pas rare qu'élite, gratin ou crème par exemple soient employés péjorativement, par anti-phrase.

On pourrait classer nébuleuse dans cette série 2, même si le terme n'est, en fait, ni positif ni négatif mais, plutôt, renvoie à quelque chose de difficile à appréhender : un référent nommé nébuleuse ou qualifié tel est vu comme intrinsèquement flou et composite.

Enfin notons qu'élite est fortement polysémique : toujours mélioratif au niveau lexical, il peut être qualifiant - il appartient alors à la série 2 -, ou être un véritable terme supposé dénommer une catégorie sociologique (voir ci-dessous série 3). Ce sens est fréquent dans les médias et les sciences sociales où, malgré le glissement sémantique, et surtout la substantialisation subreptice opérée, il ne semble pas susciter de discussion. Ainsi, l'énoncé (19) utilise le terme élite pour poser simplement l'appartenance de Cyril Ramaphosa à une catégorie.

(19) Cyril Ramaphosa fait partie de cette élite riche en Afrique du sud (radio, 9/01/2013).

Élite est donc à cheval entre les séries 2 et 3.

\section{Modes de catégorisation, noms de catégories, identité}

Les Ncoll-H de cette série sont potentiellement «sensibles", pour des raisons mentionnées plus haut : l'assignation d'un membre - une personne - à une catégorie ou à une identité se discute. En outre, si tous les noms de catégories ou d'identités ne sont pas a priori marqués péjorativement (voir francophonie, électorat, noblesse, magistrature, clergé, auxquels on ajoutera des noms de catégories politiques comme droite, gauche, 
centre ainsi que majorité, opposition), d'autres le sont systématiquement (bureaucratie, oligarchie, et souvent bourgeoisie). En bref, pour la série 3, se pose la question, sensible ou pas selon les noms et leur référent, de l'appartenance, de la catégorisation voire de l'assignation identitaire, combinée, ou pas, à une éventuelle axiologie ancrée dans le signifié même du lexème.

Voici quelques dénominations «identitaires", pour lesquels la péjoration est morphologiquement marquée: flicaille, marmaille, prêtraille, valetaille, et presque trop évidente pour que ces noms soient réellement porteurs d'enjeux.

Et quelques exemples de noms de modes de catégorisation en usage dans les sciences sociales (caste, classe, ethnie) et de catégories (aristocratie, intelligentsia, élite, jet set, nomenklatura, patriarcat, patronat, prolétariat). Comme on l'a vu déjà - $\$ 1$, exemple (9) -, caste glisse facilement du sens de 'mode de catégorisation' à celui de 'catégorie', et dans ce cas avec péjoration. Dans ce sens, il devient un parasynonyme d'oligarchie ou de tout terme désignant le pouvoir, qu'il soit économique, politique ou symbolique. Voir (20) avec la nomination qualifiante par caste :

(20) Juan Carlos Monedero, l'un des fondateurs et idéologues de Podemos : «J'ai fait ma thèse de doctorat sur l'échec de la RDA, mais personne n'en parle. Toute cette campagne menée par la "caste" vise à diaboliser le parti ", affirme-t-il. La "caste" (politique) est le grand ennemi désigné par Podemos, qui la qualifie de "majordome des riches" et insiste sur la corruption qui la ronge (Le Monde, 31/05/2014)

Pour finir, sans prétendre épuiser le sujet, citons des Ncoll-H qui renvoient à un mode de regroupement qu'on peut juger négatif ou, du moins, négociable, discutable: chapelle, école, faction, ainsi que secte déjà mentionné, tous lexèmes qui marquent la différence, l'opposition par rapport à un groupe englobant.

\section{Retour à la prédication : Noms collectifs humains attributs}

Ce qui vient d'être présenté en termes sémantiques peut être mis en lien avec la question de la prédication, ou plutôt de la prédicativité concernant les Ncoll-H. Après la prédication implicite, "contenue " dans la nomination ou dans l'apposition au sein d'un SN en fonction agentive ( $(1)$, on franchit un échelon lorsque la prédication est explicite, c'est-à-dire dans le cas des SN avec Ncoll-H en fonction attribut. Le sens de ces SN attributifs, et surtout la fonction discursive de la prédication s'apprécient à la lumière de la signification de différents Ncoll-H et des questions d'identité et d'appartenance ( $\$ 2.2$ et 2.3 ), mais aussi, inversement, les possibilités de prédications attributives et le sens qui s'en dégage permettent d'apprécier les Ncoll-H - et leur éventuel caractère problématique, discutable, sensible. C'est du moins la piste de recherche que j'emprunte, en envisageant une extrapolation à partir des exemples que j'ai pu rencontrer. Dans le cas le plus simple, celui des Ncoll-H explicitement axiologiques, on peut les mettre en relation avec les prédications qualifiantes (vs identifiantes ou classifiantes). Mais en dehors de ces cas, la situation est plus complexe.

Voici quelques exemples d'énoncés attributifs. Pour plus de lisibilité, je reprends le prédicat nominal attribut sous chaque exemple :

(21) [à propos de rependis du jihadisme, témoins dans un documentaire] Les gens qui ont témoigné sont tous menacés de mort. C'est comme une mafia : dès que l'un sort du groupe, il est considéré comme un traitre. (Télérama, 23/12/2015) 
51

Une mafia

(22) [informations, à propos de la proposition de Robert Ménard, maire de Béziers, de créer une "garde biterroise »] [...] ces vigiles, que l'opposition appelle des milices (France Culture, 16/12/2015). (association, corps solidaire, respectivement dans l'affirmation et dans la négation) sont testés, jaugés, appréciés pour leur adéquation à l'argumentation elle-même, un peu comme en (12) avec liste.

(26) L'Europe échoue aussi sur un troisième point [...] celui de la solidarité. Elle n'est à l'évidence qu'une simple association, pas un corps solidaire (interview Bertrand Badie, Télérama, 8/07/2015).

Une simple association; (pas un) corps solidaire 
ici les limites d'une différenciation stricte entre évaluation et information qualification vs classification et identification -, les deux «pôles » étant souvent mêlés, pour les raisons données en introduction : le choix des termes - ici Ncoll-H en fonction attribut - renvoie à un angle d'attaque.

Au-delà de ces exemples, on peut envisager de construire des tests à partir des Ncoll-H attributs. Cette fonction syntaxique me parait en effet susceptible d'agir comme un révélateur pour apprécier le caractère factuel ou au contraire négociable, potentiellement problématique d'un item ${ }^{22}$. On peut ainsi tester la possibilité vs la difficulté d'interprétation de tel nom dans cette fonction, et l'engagement que peut représenter la phrase en termes évaluatifs voire axiologiques. On retrouve avec les Ncoll-H en fonction attributive les possibilités d'interprétation de «est-un»: qualification, classification, identification. Compte tenu de ce qui a été développé en $\S$ 2 , on admettra que, dans le cas où les Ncoll-H sont nettement axiologiques (série 2 en $\S$ 2.3), la qualification peut être isolée des deux autres interprétations, mais qu'elle peut aussi être est compatible avec elles.

Voici à simple titre d'illustration, quelques exemples, construits à partir des Ncoll-H cités en $\S 2.3$. Je teste également ces noms avec l'enclosure une sorte de. Combiné, dans cette structure, avec des Ncoll-H, ce modalisateur a un comportement étonnant, différent selon que le Ncoll-H comporte un trait axiologique - (27d) - ou non - (27c).

61 Les exemples (27a) et (27d) portent sur la qualification : les noms qui y figurent sont évaluatifs, ce qui est renforcé par la structure attributive elle-même ; mais tous ne sont pas possibles avec l'enclosure (? une sorte d'élite;? une sorte de clique/? une sorte de canaille) - on peut penser que ces Ncoll-H sont trop nettement axiologiques. Inversement, (27b) et (27c) contiennent des Ncoll-H plutôt classifiants, supposés sans caractère évaluatif. La série (27b) peut s'interpréter en termes de classification ou d'identification (si, par exemple, $\mathrm{X}$ est un nom propre). L'enclosure une sorte de a deux effets : elle permet d'atténuer une classification trop précise - (27c) - et, en (27d), de maintenir la classification («sorte»), en combinaison avec la qualification que comportent l'emploi des Ncoll-H.

(27a) X est une canaille/ une clique/ l'élite/ une élite ; X est une caste / un lobby/ une mafia/ une oligarchie

(27b) X est une chorale/ un orchestre/ un parti/ un syndicat

(27c) X est une sorte d'association/ de club/ de mouvement/ de parti/ de réseau/ de syndicat

(27d) X est une sorte de caste/ de faction/ de gang/ de groupuscule/ de junte/ de lobby/de mafia/ de milice/ d'oligarchie/ de phalange.

Enfin, certains Ncoll-H présentent peu de négociabilité, et, dans ce cas, la structure attributive paraît difficile : que dire de «X est une magistrature/ un électorat »? La phrase paraît tautologique, absurde.

\section{En conclusion}

«Les mots sont importants »: la remarque apparait sur la scène publique comme un véritable lieu commun; en tout état de cause, elle dénote la sensibilité des acteurs sociaux à l'enjeu du langage, lorsqu'il s'agit de qualifier, de représenter, de catégoriser, de nommer.

Argumentation et Analyse du Discours, 17 | 2016 
64

Dans la perspective de ces débats, les Ncoll-H constituent un objet linguistique remarquable : leur sens est composite, comportant deux niveaux - celui des éléments et celui du groupe unitaire -, de sorte que l'articulation de ces deux niveaux comporte intrinsèquement une part de complexité et de négociabilité. Sur un autre plan, comme dénomination de groupes humains, ils ont leur place sur la scène des affaires humaines, dans leur complexité - questions de regroupement et d'association, d'affiliation et d'alliance, d'appartenance et d'identité.

En partant d'observations sur les Ncoll-H, j'ai cherché à tester le rapprochement qu'on peut établir à leur propos entre nomination et prédication - ce qui revient finalement à se demander ce qui est "contenu» dans tel nom, en termes de qualification, de catégorisation ou d'identification. Certains Ncoll-H, explicitement évaluatifs, présentent une affinité évidente avec la prédication, spécifiée en qualification. Mais pour d'autres, la question peut déboucher sur des propriétés plus souterraines de la nomination, et donc de représentation du collectif. Quoi qu'il en soit, l'approche me paraît en définitive fructueuse parce qu'elle permet de révéler, à propos de cette catégorie spécifique de noms, la part d'implicite, de présupposé, d'implication du sujet ou de la collectivité dans l'emploi d'une expression nominale dans le but de référer.

\section{BIBLIOGRAPHIE}

Amossy, Ruth. 2000. L'argumentation dans le discours. Discours politique, littérature d'idées, fiction (Paris : Nathan)

Amossy, Ruth \& Roselyne Koren. 2004. « Présentation », Semen 17, 9-18

Charolles, Michel. 2002. La référence et les expressions référentielles en français (Gap : Ophrys)

Détrie, Catherine, Paul Siblot et Bertrand Verine. 2001. Termes et concepts pour l'analyse du discours : une approche praxématique (Paris : Honoré Champion)

Frath, Peter. 2010. «La référence par le nom : vers une linguistique anthropologique », Peter Frath, Laure Lansari \& Jean Pauchard (éds).Res per Nomen 2 : langue, référence et anthropologie (Reims, EPURE). En ligne http://www.res-per-nomen.org/index.html

Gary-Prieur, Marie-Noëlle. 1998. « Prédication et référence : quand un prédicat devient désignateur, et inversement », Forsgren, Mats, Kerstin Jonasson \& Hans Kronning (éds).

Prédication, assertion, information (Uppsala : Acta Universitatis Upsaliensis),201-209

Kerbrat-Orecchioni, Catherine. 2014 [1980]. L'Énonciation. De la subjectivité dans le langage (Paris : Colin)

Kleiber, Georges. 2001. « Remarques sur la dénomination », Cahiers de praxématique 36, 21-41

Lammert, Marie. 2010. Sémantique et cognition : les noms collectifs (Genève-Paris : Droz)

Lammert, Marie \& Michelle Lecolle. 2014. « Les noms collectifs en français, une vue d'ensemble », Cahiers de Lexicologie 105, 203-222 
Lecolle, Michelle. 2007. « Enjeux argumentatifs de la nomination : le cas du nom collectif communauté dans les discours publics contemporains », Christian Boix (éd.). Argumentation, manipulation, persuasion (Paris : L'Harmattan), 227-247

Lecolle, Michelle. 2013. « Noms collectifs humains : un point de vue de sémantique lexicale sur l'identité dans le rapport individu/groupe ». Revue ¿Interrogations? 16 [en ligne]

Milner, Jean-Claude. 1978. De la syntaxe à l'interprétation. Quantités, insultes, exclamations. (Paris : Seuil)

Perelman, Chaim \& Lucie Olbrechts-Tyteca. 1970 [1958]. Traité de l'argumentation. La nouvelle rhétorique (Bruxelles : Éditions de l'Université de Bruxelles)

Reichler-Béguelin, Marie-José. 1995. « Alternatives et décisions lexicales dans l'emploi des expressions démonstratives ", Pratiques 85, 53-87

Reichler-Béguelin, Marie-José. 1998. « Le statut des informations non identifiantes dans le SN défini anaphorique », Forsgren, Mats, Kerstin Jonasson \& Hans Kronning (éds). Prédication, assertion, information (Uppsala : Acta Universitatis Upsaliensis), 463-474

Ricœur, Paul. 1975. La métaphore vive (Paris : Seuil)

Schnedecker, Catherine. 2006. «SN démonstratifs “prédicatifs” : qu'est-ce qui limite leur apport informatif? », Langue Française 152, 39-55

Sériot, Patrick. 1986. «Langue russe et discours politique soviétique : analyse des nominalisations ", Langages 81, 11-41

Sériot, Patrick. 1993. " La grande partition (enchâssement syntaxique, stratification énonciative et mémoire du texte) ». Patrick Sériot (éd.), Relations inter- et intra-prédicatives, Cahiers de l'ILSL 3, 235-260

Siblot, Paul. 2001. « De la dénomination à la nomination. Les dynamiques de la signifiance nominale et le propre du nom », Cahiers de praxématique 36, 189-214

Van Peteghem, Marlène. 1998. «Sur le contenu lexical des descriptions définies et démonstratives », Forsgren, Mats, Kerstin Jonasson \& Hans Kronning (éds). Prédication, assertion, information (Uppsala : Acta Universitatis Upsaliensis), 569-578

Wiederspiel, Brigitte. 1995. «Sur quelques aspects de la saisie démonstrative », Pratiques 85, 113-125

\section{NOTES}

1. Pour une discussion sur l'argumentation par la nomination avec le Ncoll communauté, voir Lecolle (2007).

2. Il ne s'agit pas à proprement parler d'un corpus, mais d'une collecte systématique d'occurrences de Ncoll-H dans la presse papier et en ligne de tous genres, et parfois la radio, essentiellement depuis les années 2010. Plus anciennement, mes collectes se sont centrées sur certains Ncoll-H (communauté notamment).

3. Conception proche de celle qu'on trouve chez Siblot (2001), et dans Détrie et al. (2001), article «nomination», par le même auteur. Je laisse de côté le cas du nom propre, pour lequel la qualification « pourvu de sens »- qui me parait importante - nécessiterait discussion.

4. Comme la nomination, la référence est un acte de langage; les deux termes sont des quasisynonymes, mais ne sont pas employés indifféremment, selon les champs théoriques. Par 
ailleurs, la référence peut concerner différentes parties du discours, alors que la nomination privilégie les expressions nominales.

5. Voir l'entrée "prédication » dans Détrie et al. (2001). Et voir la célèbre étude de Sériot luimême (1986) sur les nominalisations en russe dans le discours politique soviétique.

6. On nomme "anaphore" un procédé consistant à rappeler un mot ou groupe de mots précédemment énoncé. Ce faisant, l'anaphore (ré-)oriente la présentation du référent - voir différents cas et illustrations plus loin.

7. Anaphore constituée d'un SN débutant par un article défini : «la cinéaste » reprenant Agnieszka Holland en (1).

8. Le SN anaphorique débute par un déterminant démonstratif: «cet absent» en (3), «ce mouvement » en (4).

9. Voir Van Peteghem (1998) et les références qu'elle cite.

10. Les exemples de (7a) et (8a) contiennent des noms de qualité (NQ), et sont empruntés aux analyses de Milner (1978). Je les mets en regard de Ncoll-H évaluatifs tels que lie, dans le but de montrer le parallèle de comportement, du point de vue qui m'occupe ici.

11. http://www.anonymes-france.eu/, consulté le $1^{\mathrm{er}}$ septembre 2014.

12. Je me concentre sur les nominations, et du seul point de vue qui m'occupe. Mais naturellement, les phrases de l'exemple présentent aussi des enchainements argumentatifs, dans lesquels ces nominations jouent un rôle, notamment comme jalons de la construction d'une opposition.

13. Selon le Petit Robert (1994), nomenklatura « (pays de l'Est) : liste de personnes bénéficiant de prérogatives exceptionnelles; par extension, ensemble de personnes privilégiées ». Est-on ici dans le cas de « par extension »? Peu importe en définitive, le raisonnement reste le même.

14. On pourrait tout aussi bien dire «la nomination».

15. Voir Lecolle $(2007,2013)$ pour des développements plus conséquents.

16. C'est-à-dire, en clair, d'origine étrangère, et plus précisément des anciennes colonies françaises. Ce nom morphologiquement construit sur « divers»n'a pas le sens que laisse prévoir la dérivation - voir (14). Il nécessiterait une analyse lexico-sémantique plus complète. Dans l'espace public français, minorités visibles (au pluriel) dénote sensiblement la même « réalité » que le Ncoll-H diversité - sauf qu'on ne peut pas réellement en être certain, car cela dépend du locuteur (pour certains, minorité visible englobe les femmes).

17. Ce qui ne veut pas dire qu'ils soient toujours repérés comme tels. Qui mentionne le caractère problématique (selon moi) de diversité, dans l'acception relevée ci-dessus? De fait, cette dénomination appartient au bagage contemporain des lieux communs (au sens ordinaire du terme).

18. Pour les notions d'évaluation et d'axiologie, voir Kerbrat-Orecchioni (2014). Je recours à la notion d'axiologie lorsque l'évaluation est de type bon/mauvais, bien/mal.

19. Faute de place, je ne présente pas de manière circonstanciée le fondement de mes catégories de Ncoll-H. Le mode de rassemblement des éléments (fonctionnel, identitaire notamment) y joue un rôle fondamental.

20. Voir Kerbrat-Orecchioni (2014) pour une discussion sur les rapports entre évaluation axiologique dans les valeurs liées au référent et au niveau du signifié.

21. Dans mes relevés d'exemples, nébuleuse apparait en fonction attribut ou plus généralement prédicative davantage que dans des $\mathrm{SN}$ à fonction référentielle.

22. Ce qui n'épuise pas, comme on l'a vu avec diversité, la question plus générale des enjeux de la nomination par un Ncoll-H. Et qui, par ailleurs, présente aussi toutes les difficultés liées à la polysémie. 


\section{RÉSUMÉS}

L'article porte sur la nomination collective d'êtres humains : il s'agit, plus précisément, d'aborder la sémantique des noms collectifs humains (Ncoll-H), sous l'angle d'une dimension évaluative ou prédicative potentielle. On observe, à propos des Ncoll-H, cette dimension prédicative sous différents aspects : elle peut être liée à la sémantique du nom collectif même, aux traits lexicaux de telle série de Ncoll-H ou à leurs emplois préférentiels. Une première partie explore le rapprochement entre nomination et prédication. Dans une deuxième partie, on présente les propriétés sémantiques des Ncoll-H et la signification lexicale de certains, en rapport avec leurs potentialités prédicatives ou évaluatives. Une dernière partie aborde les emplois attributifs de Ncoll-H. Cette étude permet de situer les « enjeux socio-politiques » de la nomination de groupes humains dans le cadre d'une analyse sémantique et discursive plus générale.

This paper addresses the issue of the collective naming of human beings: more precisely, we deal with human collective nouns (Ncoll-H) in their potential evaluative or predicative dimension. Concerning the Ncoll-H, we observe this predicative dimension from different angles: it can be linked to the semantic dimension of collective nouns, to the lexical feature of one or another kind of Ncoll-H, or to the way in which they are most often employed. We first explore the connection that can be made between nomination and predication. In a second part, we introduce the semantic properties of the Ncoll-H and the lexical meaning which can be attributed to some of them, in connection with their predicative or evaluative potential. The last part addresses the attributive uses of some Ncoll-H. In this research, we aim to situate the "Sociopolitical stake" involved in the attributing of names to groups of humans in the wider framework of a more generic semantic and discursive analysis.

\section{INDEX}

Mots-clés : identité, nom collectif humain, pluralité, prédication

Keywords : human collective noun, identity, plurality, predication

\section{AUTEUR}

\section{MICHELLE LECOLLE}

Université de Lorraine, CREM 